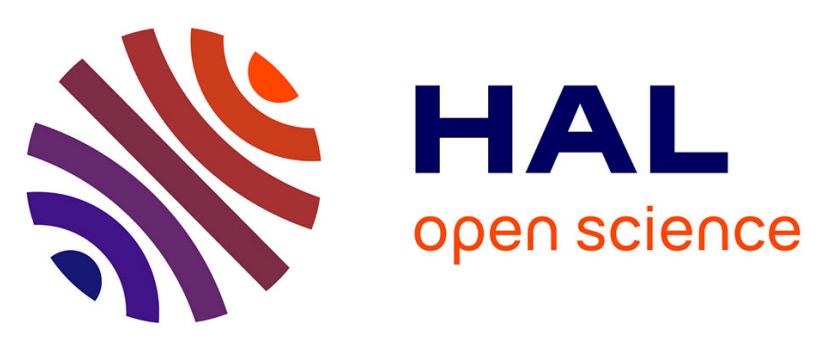

\title{
Study on Feasibility of Minority Carrier Complete Drag in Silicon. New Investigation Method Intended for Indirect-Gap Semiconductors
}

T. Mnatsakanov, L. Pomortseva, V. Shuman

\section{- To cite this version:}

T. Mnatsakanov, L. Pomortseva, V. Shuman. Study on Feasibility of Minority Carrier Complete Drag in Silicon. New Investigation Method Intended for Indirect-Gap Semiconductors. Journal de Physique IV Proceedings, 1996, 06 (C3), pp.C3-81-C3-86. 10.1051/jp4:1996312 . jpa-00254230

HAL Id: jpa-00254230

https://hal.science/jpa-00254230

Submitted on 1 Jan 1996

HAL is a multi-disciplinary open access archive for the deposit and dissemination of scientific research documents, whether they are published or not. The documents may come from teaching and research institutions in France or abroad, or from public or private research centers.
L'archive ouverte pluridisciplinaire HAL, est destinée au dépôt et à la diffusion de documents scientifiques de niveau recherche, publiés ou non, émanant des établissements d'enseignement et de recherche français ou étrangers, des laboratoires publics ou privés. 


\title{
Study on Feasibility of Minority Carrier Complete Drag in Silicon. New Investigation Method Intended for Indirect-Gap Semiconductors
}

\author{
T.T. Mnatsakanov, L.I. Pomortseva and V.B. Shuman* \\ All-Russian Electrotechnical Institute, Krasnokazarmennaya str. 12, 111250 Moscow, Russia \\ * A.F. Ioffe Physico-Technical Institute, Politechnicheskaya str. 26, 194021 St.-Petersburg, Russia
}

\begin{abstract}
A method is proposed for determining the electron - hole scattering parameters in indirect gap semiconductors when the carrier injection level is low. The proposed method is used to study the electron - hole scattering in silicon. The results are evidence that minority carrier complete drag by majority-electrons is possible in $\mathrm{n}$-type material at a doping level of $\mathrm{N}_{d}>10^{17} \mathrm{~cm}^{-3}$ even at room temperatures.
\end{abstract}

\section{INTRODUCTION}

Correct treatment of physical phenomena governing charge carrier transport in semiconductor samples allows better understanding of semiconductor device operation. Among scattering mechanisms that are responsible for observed values of charge carrier mobility in semiconductors the contribution of electronhole scattering (EHS) proves to be rather ambiguous. Since the first mention of EHS as a factor that could substantially change the charge carrier transport coefficients [1], a large number of papers devoted to the study of this phenomenon in various semiconductor materials (Ge, $\mathrm{Si}, \mathrm{GaAs}$ ) [2-15] as well as to determination of its effect on the characteristics of multilayer semiconductor devices [16-20] have appeared in the literature. It was assumed at first that EHS makes the greatest contribution to charge carrier transport under high injection conditions, when the concentration of injected carriers exceeds $10^{16}$ $\mathrm{cm}^{-3}$. Techniques for determining the parameters that characterize EHS were developed in accordance with this viewpoint and these parameters were experimentally studied under high injection conditions [6,7]. It later became clear, however, that the influence of EHS can show up much more clearly under low injection conditions. The experimental detection $[12,13]$ of the effect of minority carrier complete drag in $\mathrm{GaAs}$ confirmed the results of the theoretical treatment $[3,5]$ and stimulated interest in the possibility that this effect could appear in $\mathrm{Si}$, which is the most widely used material of semiconductor electronics. In this connection it should also be noted that a comparative estimate carried out in [21], using the experimental results of $[12,13]$, showed that effectiveness of EHS in $\mathrm{Si}$ is $40-50$ times greater than in GaAs. However up to now the minority carrier complete drag has not been detected in $\mathrm{Si}$. To our mind difficulty of studying EHS in $\mathrm{Si}$ is that, in contrast with GaAs, silicon is an indirect-gap semiconductor. This makes it difficult to use the optical techniques that directly made it possible to detect the complete drag of minority carriers by majority carriers in GaAs $[12,13]$. Thus the goal of this paper is to develop and put into practice a method for determining the EHS parameters under low injection conditions in indirect-gap semiconductors. Since our studies are based on minority carrier transport equations suggested recently $[10,14]$, the paper is organized as follows. In section 2 the newly derived minority carrier transport equations are presented, and temperature dependence of minority carrier mobility in Si is discussed. In section 3 the new method for determining parameters of EHS under low injection conditions is described. In section 4 experimental results obtained for $\mathrm{Si}$ are presented. Finally in section 5 we discuss the results. 


\section{MINORITY CARRIER MOBUITY MODEL}

According to $[10,14]$ the minority-hole current in $\mathbf{n}$ - type sample can be written as

$$
j_{p}=q p \mu_{p} \frac{\mu_{p}-\mu_{n}}{\mu_{p m}+\mu_{p}} E-q D_{p} \frac{\mu_{p}}{\mu_{p}+\mu_{p}} \frac{\phi p}{d x}
$$

where $p$ is the concentration of holes, $E$ is the electric field, mobilities $\mu_{n}$ and $\mu_{p}$ are usual mobilities of the particles obtained if EHS is ignored, $\mu_{p n}$ is mobility for which EHS is responsible. It follows from (1) that effective minority-hole mobility $\mu_{p}^{m i}$ and diffusion coefficient $D_{p}^{m i}$

are related to each other

$$
\mu_{p}^{m i}=\mu_{p} \frac{\mu_{p n}-\mu_{n}}{\mu_{p n}+\mu_{p}} \quad, \quad D_{p}^{m i}=D_{p} \frac{\mu_{p n}}{\mu_{p n}+\mu_{p}}
$$

$$
D_{p}^{m i}=\frac{k T}{q} \frac{\mu_{p m}}{\mu_{p n}-\mu_{n}} \mu_{p}^{m i}
$$

Similar formula can be obtained for minority electron mobility and diffusion coefficient in $p$-type material, by replacement of indices $n \rightarrow p, \quad p \rightarrow n$. It is worth mentioning that just recently the abovementioned equations have been repeated by Kane and Swanson [22,23] (see also [24]).

Relation (3) differs from Einstein relation in nondegenerated semiconductors. The reason for violation of Einstein relation has been thoroughly investigated in [10]. It consist in the fact that in formula (1) the components representing the drag current are formally combined with diffusion and drift components.

Relation (3) proves to be of great importance for interpretation of current experimental data on minority carrier mobility in semiconductors. The reason is that modern experimental methods used at present consist in measuring the minority carrier diffusion coefficient and then calculating the mobility value with the help of Einstein relation. Since formula (3) shows that EHS changes the relationship between minority carrier mobility and diffusion coefficient, now it is quite evident that experimental results presented in literature $[25,26]$ must be recalculated. This recalculation involves certain difficulty conditioned by ambiguity of $\mu_{p n}$ value under low injection conditions. One possible way to overcome this difficulty consists in utilization of following formula $[14,15]$ :

$$
\mu_{p m}=\frac{G}{\frac{n}{n_{0}}+\frac{n / n_{2}}{1+(n+p) / 2 n_{1}}}
$$

where $G=1840 \mathrm{~cm}^{2} / \mathrm{V} / \mathrm{s}, \quad \mathbf{n}_{0}=3.210^{17} \mathrm{~cm}^{-3}, \mathbf{n}_{1}=3.510^{16} \mathrm{~cm}^{-3}, \quad \mathbf{n}_{2}=4.610^{16} \mathrm{~cm}^{-3}$.

According to [20] this formula is just a result of extrapolation of experimental data obtained by Dannhauser [6] and Krausse [7] to low injection conditions.

Incorporation of the formula (3) into analysis of current experimental data on minority carrier mobility results in two main conclusions. Firstly the minority carrier mobility may not exceed the majority carrier mobility $[14,15]$. Secondly the minority carrier mobility values in highly doped samples can be fitted with a positive power law dependence at temperature $T \leq 300 \mathrm{~K}$

$$
\mu^{m i} \propto T^{+n}
$$

In Fig. 1 an example of recalculation of experimental data using formula (3) is presented. In Fig. 1 solid line 1 corresponds to data presented in [25] for doping density $N=5.310^{18} \mathrm{~cm}^{-3}$. After recalculation it converts into curve 2 , which can be fitted with positive power law dependence at $T \leq 300 \mathrm{~K}$.

Dependence (5) completely contradicts to commonly accepted one $[25,26]$. However to confirm it we need experimentally justified values of $\mu_{p n}$ under low injection conditions, since it is well known that extrapolation procedure may lead to ambiguous result. In section 5 we shall discuss this point on the basis of experimental results obtained in section 4. 


\section{METHOD FOR DETERMINING EHS PARAMETERS}

Since optical methods are inapplicable in indirect-gap semiconductors, we shall try to investigate the EHS parameters in Si by measuring the characteristics of multilayer structure that depend on EHS, after which the EHS parameters will be determined by calculation. More particularly our method is based on investigation of current-voltage characteristics (CVC) of $p^{+}-n-n^{+}$diode structure. Our choice is based on the results obtained in [15], which show that EHS has a strong effect on static CVC of $p-n$ junction. Using transport equations that correspond to the low injection conditions in $n$-base layer and taking into account that that the dopant distribution in $n$-base is homogeneous, it is easy to show that continuity equation in diffusive approximation can be reduced to

$$
\frac{d^{2} p}{d x^{2}}-\frac{p}{L_{p}^{2}}=0
$$

where $L_{p}=\left(D_{p} \frac{\mu_{p n}}{\mu_{p n}+\mu_{n}} \tau_{p}\right)^{1 / 2}, D_{p}$ and $\mu_{p}$ are the usual hole diffusion coefficient and mobility respectively and $\tau_{p}$ is the hole lifetime.

Since the region of small current densities is of interest for us, it is natural to take the condition that the injection efficiency of the junction equals unity as a boundary condition on the injecting contacts. Thus we can write that

$$
\left.j_{p}\right|_{x=0}=j,\left.\quad j_{n}\right|_{x=W_{n}}=j
$$

It was assumed when writing the boundary conditions that $p^{+}-n$ junction is at the point $x=0$, while the $n^{+}-n$ junction is at the point $x=W_{n}$ :

The solution of equation (6), with boundary conditions (7), is perfectly obvious; therefore without dwelling on the calculations, we shall present and discuss the final results.

Standard computations result in an CVC characteristics described by

$$
V=\frac{k_{B} T}{q} \ln \left(\frac{j}{j_{0}}\right)+j \rho_{n} W_{n}\left[1+\frac{b-1+2 b\left(\mu_{p} / \mu_{p n}\right)}{\left(W_{n} / L_{p}\right)}\left(1-\exp \left(-\frac{W_{n}}{L_{p}}\right)\right)\right]
$$

where $V$ is voltage applied to the structure, $\rho_{n}$ is the base layer resistivity, $k_{B}$ is Boltzmann constant, $T$ is the temperature, $p_{0}$ is the equilibrium hole concentration in the $n$ base $b=\mu_{n} / \mu_{p}$ and $j_{0}$ is defined by

$$
j_{0}=\frac{q D_{p} p_{0}}{L_{p}}\left(\frac{\mu_{p n}}{\mu_{p m}+\mu_{p}}\right) \frac{\sinh \left(W_{n} / L_{p}\right)}{\cosh \left(W_{n} / L_{p}\right)}
$$

The obtained CVC depends upon the mobility $\mu_{m}$ and can therefore be used to determine it. Unfortunately it is hard to obtain an expression in general for $\mu_{p n}$ from equation (8), and therefore we consider the limiting case, which is convenient for studying structures with a high base-layer doping level. The charge carrier lifetime decreases as the doping level increases or the temperature of the structure decreases, which results in an increase of the ratio $\left(W_{n} / L_{p}\right)$. When $\left(W_{n} / L_{p}\right) \geq 5$, the ratio of hyperbolic functions is close to unity. As a result the expression for $j_{0}$ simplifies to

$$
j_{0}=q\left(\frac{D_{p} \mu_{p m}}{\tau_{p}\left(\mu_{p m}+\mu_{p}\right)}\right)^{1 / 2} p_{0}
$$


When the base-layer doping $N_{d}$ does not exceed $10^{17} \mathrm{~cm}^{-3}$, the equilibrium hole concentration can be written as $p_{0}=n_{i 0}^{2} / N_{d}$, where $n_{i 0}$ is the intrinsic carrier concentration in a weakly doped sample of the material. We can then obtain the following expression for $\mu_{m}$ from equation (8)

$$
\mu_{p n}=\frac{\mu_{p}}{A^{2}-1}
$$

where $\quad A=\left(\frac{q n_{i 0}^{2}}{j N_{d}}\right)\left(\frac{D_{p}}{\tau_{p}}\right)^{1 / 2} \exp \left(\frac{q\left(V-j \rho_{n} W_{n}\right)}{k_{B} T}\right)$.

When $N_{d} \geq 10^{17} \mathrm{~cm}^{-3}$, the narrowing of the silicon band-gap should be taken into account. To do this we use the data presented in paper [27] and take into account the corrections introduced into experimental technique of this paper by electron-hole collisions [28]. We obtain then the following expression for $\mu_{p m}$

$$
\mu_{p n}=\frac{\mu_{p}}{B^{2}-1}
$$

where $B=\left[\frac{q n_{i 0}^{2} \exp \left(\Delta E_{g} / k_{B} T\right)}{j N_{d}}\left(\frac{D_{\underline{p}}}{\tau_{p}}\right)^{1 / 2} \exp \left(\frac{q\left(V-j \rho_{n} W_{n}\right)}{k_{B} T}\right)\right]^{-1}$.

Formulas (11( and (12) are the basis of the proposed technique for determining $\mu_{p n}$ using diode structures; $j, V, \rho_{n}, W_{n}, \tau_{p}$ and $T$ are the measured parameters, and the quantities $D_{p}$ and $\Delta E_{g}$ are taken from independent experiment. As a result, $\mu_{p n}$ can be measured directly under low injection conditions.

\section{DETERMINING THE MOBLITY $\mu_{p n}$ IN Si UNDER LOW INJECTION CONDITIONS}

We had $p^{+}-n-n^{+}$structures fabricated. The base layer doping was $N_{d}>10^{17} \mathrm{~cm}^{-3}$. The CVC was measured for each of these structures using the standard techniques. A current density region was chosen for the measurement, for which a low injection level of nonequilibrium charge carriers was produced in the base layer of the structure. The experimentally measured dependence of $j$ on $V$ was approximated by dependence like that of equation (8) and experimental value of parameter $\left(j_{0}\right)_{\text {exp }}$ was determined. A calculated value of the same parameter was then determined from

$$
\left(j_{0}\right)_{c a l}=\frac{q n_{i 0}^{2} \exp \left(\Delta E_{g} / k_{B} T\right)}{N_{d}}\left(\frac{D_{p}}{\tau_{p}}\right)^{1 / 2}
$$

Then taking into account that $B=\left(j_{0}\right)_{\exp } /\left(j_{0}\right)_{c a l}$ the value of mobility $\mu_{p n}$ was determined utilizing formula (12). The final results of our investigation are presented in Fig. 2 (see curve 3) with allowance made for scatter of electrophysical parameter values. It is worth mentioning that because of the complexity of determining the lifetime $\tau_{p}$ in highly doped samples, our calculation allows for the scatter of the values of this parameter indicated in the literature, e.g. according to the data presented in review paper [29] for $N_{d}=2.310^{17} \mathrm{~cm}^{-3}$ parameter $\tau_{p}$ lies in the interval $2 \mu s \leq \tau_{p} \leq 16 \mu \mathrm{s}$. O course this leads to a rather large scatter of $\mu_{p m}$ values, however we shall show that even these results are extremely useful for describing the properties of minority charge carriers in Si. Besides new experimental results Fig. 2 also shows the results of extrapolation of the data for $\mu_{p n}$ in accordance with formula (4) (see curve 1). It can be seen that the measured $\mu_{p m}$ values, first, turn out to be substantially smaller than the extrapolated values, and, second. they lie below curve 2 , which represents the dependence on $N_{d}$ of the mobility of the majority carriers, which are electrons (the scatter of the electron mobility is shown in accordance with 
the data presented in [30]). The latter circumstance is of special interest, since according to formula (2) (see also $[14,15]$ ) the condition $\mu_{p r}<\mu_{n}$ is evidence that minority-holes complete drag by majority electrons is possible.

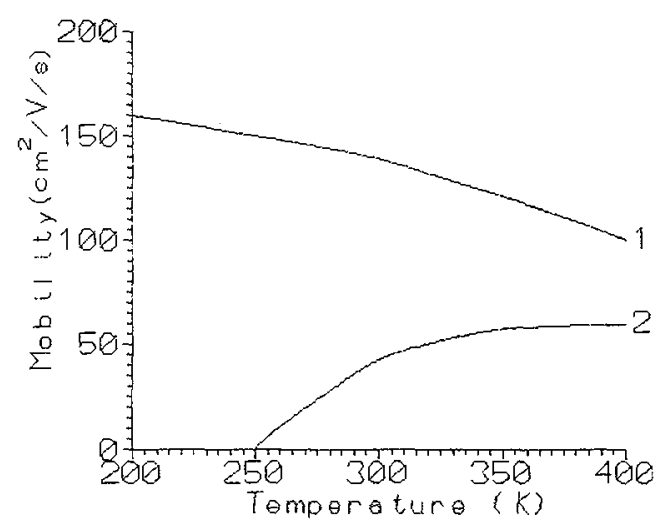

Figure 1: The temperature dependence of minority electron mobility for doping density $N=5.310^{18} \mathrm{~cm}^{-3 /}$

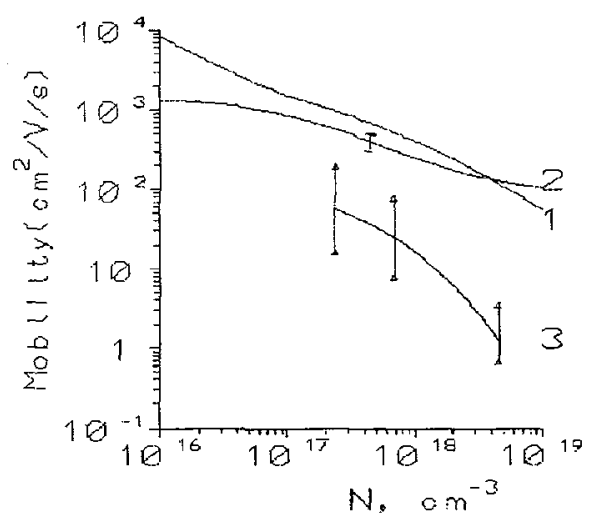

Figure 2: Mobility $\mu_{p n}$ dependence on doping concentration under low injection conditions

\section{DISCUSSION OF THE RESULTS}

We have already mentioned that minority carriers complete drag by majority carriers was experimentally detected in GaAs at a temperature of ' $T \approx 90 K[12,13]$ The estimates presented in [21] showed that effectiveness of EHS in $\mathrm{Si}$ is 40-50 times greater than in GaAs. However, until now the possibility implementing this effect in Si has not even been considered in the literature. In spite of the significant error of the experimental values, the results of this paper show that the condition $\mu_{p n}<\mu_{n}$ is satisfied in $\mathrm{Si}$ even at room temperature $T=21^{0} \mathrm{C}$ in the dopant-concentration region $N_{d}>10^{17} \mathrm{~cm}^{-3}$, i.e. minority carriers complete drag is possible in Si under these conditions.

Turning back to temperature dependence of minority carrier mobility we can argue now that formula (5) holds since effectiveness of EHS proves to be even greater than it follows from extrapolated formula (4). In conclusion we should point out that the main error in the values of mobility $\mu_{p n}$ is determined by the scatter of the hole lifetime $\tau_{p}$. Improving the techniques for determining $\tau_{p}$ will make it possible to substantially enhance the accuracy with which $\mu_{p n}$ is determined.

\section{Acknowledgements}

This work was supported by INTAS project No. 94-0417. 


\section{References}

[1] Fletcher N.H.,Prog.IRE 6 (1957) 862-872.

[2] Paige E.G.S., Physics and Chemistry of Solids 16 (1960) 207-219.

[3] McLean T.P. , Paige E.G.S., J. Phys. Chem. Solid 16 (1960) 220-236.

[4] Davies L.V., Nature 194 (1962) 762-763.

[5] Gribnikov Z.S., Mel'nikov V.I., Sov.Phys. Semicond. 2 (1968) 1133-1143.

[6] Dannhauser F., Solid-State Electron. 15 (1972) 1371-1375.

[7] Krausse J. R., Solid-State Electron. 15 (1972) 1376-1381.

[8] Mnatsakanov T.T., Rostovtsev I.L., Filatov N.I.,Sov.Phys.Semicond. 18 (1984) 807-809.

[9] Dumke W.P., Solid-State Electron. 28 (1985) 183-186.

[10] Mnatsakanov T.T., Phys. Status Solidi 1143 (1987) 225-234.

[11] Hansch W., Mahan G.D., Physics and Chemistry of Solids 44 (1983) 663-670.

[12] Hopfel R.A., Shah J.,Wolff P.A., Gossard A.C., Phys.Rev.Lett. 56 (1986) 2736-2739.

[13] Hopfel R.A., Shah J.,Wolff P.A., Gossard A.C., Appl.Phys.Lett. 49 (1986) 572-574.

[14] Gresserov B.N., Muatsakanov T.T., Sov.Phys.Semicond. 23 (1989) 1025-1028.

[15] Mnatsakanov T.T., Gresserov B.N., Pomortseva L.I., Solid-State Electron. 38 (1995) 225-233.

[16] Kokosa R.A., Proc.IEEE 55 (1967) 1389-1400.

[17] Grekhov I.V., Otblesk A.E., Radiotekh. Electron. 19 (1974) 1489-1490.

[18] Adler M.S., IEEE Trans.Electron. Devices 25 (1978) 16-22.

[19] Gresserov B.N., Mnatsakanov T.T., Sov. Journal Tech. Phys. 31 (1986) 1090-1091

[20] Mnatsakanov T.T., Rostovtsev I.L., Filatov N.I., Solid-State Electron. 30 (1987) 579-586.

[21] Gresserov B.N., Mnatsakanov T.T., Sov.Phys.Semicond. 24 (1990) 1042-1043.

[22] Kane D.E., Swanson R.M., J. Appl. Phys. 72 (1992) 5294-5304.

[23] Kane D.E., Swanson R.M., IEEE Trans. Electr. Dev. 40 (1993) 1496-1500.

[24] Mnatsakanov T. T., IEEE Trans. Electr. Dev. 42 (1995) 2251-2253.

[25] Swirhun S.E., Kane D.E., Swanson D.E., "Temperature Dependence of Minority Electron Mobility and Bandgap Narrowing in $\mathrm{P}^{+} \mathrm{Si}^{\prime}$, IEEE Electron Device Meeting, USA 1996, pp. 298-301.

[26] Wang C.H. Misiakos K., Neugroschel A., Appl. Phys. Lett. 57(1990) 159-161.

[27] Slotboom J.W, deGraaff H.C., Solid-State Electron. 19 (1976) 857-863.

[28] Mnatsakanov T.T., Pomortseva L.I., Yakovlev D.G., Sov. Phys. Semicond. 28 (1994) 1059-1062.

[29] Fossum J.G., Mertens R.P., Lee D.S., Nijs J.F., Solid-State Electron. 26(1983)569-576.

[30] Jacoboni C., Canali C., Ottaviani G., Quaranta A., Solid-State Electron. 20 (1977) 77-89. 